\title{
RESILIENCE SHIFT PROGRAM: OBJECTIVES, EXECUTION AND PRELIMINARY RESULTS
}

\author{
Jan Reier Huse ${ }^{1}$ \\ Oliver Pritchard, Juliet Mian, Xavier Aldea Borruel ${ }^{2}$ \\ ${ }^{1)}$ Lloyd's Register Foundation, London, UK \\ ${ }^{2)}$ Resilience Shift, London, UK
}

\begin{abstract}
The Resilience Shift (RS) was established in 2016 to address the recommendations of the Lloyd's Register Foundation's 'Foresight review of resilience engineering'. The initial 5-year programme is funded by Lloyd's Register Foundation, with Arup as host institution. Its aim is to inspire and empower a shift in critical infrastructure resilience thinking and practice so that engineered structures and infrastructure will be not only safer but also better. This paper provides a summary of the Resilience Shift's aims and objectives and explains the programme execution. Building resilience of critical infrastructure requires decisionmakers working in different industry sectors to understand 'what' can be done, 'why' it should be done, and 'how' to put it into practice. All results from the Resilience Shift programme are publicly available through the organisation's website, which can be accessed at:

https://www.resilienceshift.org/.
\end{abstract}

Keywords: Resilience, Critical infrastructure systems, value chain, socio-technical systems

\section{INTRODUCTION}

The safety and wellbeing of billions of people depends on infrastructure systems that can deliver critical services that can provide, protect or connect us; whatever the future has in store. However, the future isn't what it used to be, and rising populations, increasing urbanization and globalization alongside climate change are putting extreme pressures on our infrastructure systems. The World Economic Forum recently identified the 'failure of critical infrastructure’ as a key global economic risk [1].

Resilience building allows you to prevent or mitigate against shocks and stresses you identify and be better able to absorb, respond and recover, and adapt to those you can't predict or avoid [2]. Resilience requires some understanding and consideration of complexity and the interdependence of systems and services underpinning modern life.

This paper introduces the Resilience Shift ${ }^{1}$, which is aiming to improve the resilience within and between critical infrastructure. We discuss the origins of the Resilience Shift and share its journey to date.

\footnotetext{
${ }^{1}$ https://www.resilienceshift.org/

(C) 2019 Authors. This is an Open Access article distributed under the terms of the Creative Commons AttributionNonCommercial 4.0 International License (http://creativecommons.org/licenses/by-nc/4.0), permitting all non-commercial use, distribution, and reproduction in any medium, provided the original work is properly cited.

ISBN: 978-91-88898-41-8
}

DOI: https://doi.org/10.15626/rea8.04 


\section{THE RESILIENCE SHIFT}

\subsection{Beginnings}

The Resilience Shift was set up in 2016, for an initial 5-year period, to address the recommendations set out in the Lloyd's Register Foundation's (LRF) 'Foresight review of resilience engineering' [3] to improve the resilience of critical infrastructure.

Following an open competition, Arup ${ }^{2}$ was awarded the contract to manage the Resilience Shift programme on behalf of LRF; the programme has been provided with 10 million GBP funding for this initial 5-year period. Arup has been appointed as host institution; programme delivery activities are performed in collaboration with a network of grantees.

Some activities are co-funded with other organizations. Organisations involved in the delivery of different programme activities are detailed on the Resilience Shift website and comprise a mixture of different types of organisations, including academia, industry and think tanks.

\subsection{Lloyd's Register Foundation}

Lloyds Register was founded in 1760 and is one of the earliest global businesses. Its primary operations are towards the marine, energy and transport sectors. The businesses focus is on safety, environment and quality. LRF is the sole owner of Lloyd's Register Group and is a charity trust that supports safety of life and property and public education. It is a UK headquartered organisation, and funds projects worldwide. LRF is 'Engineering a safer world, because life matters', focusing on safety and education.

The 2015 LRF report 'Foresight review of resilience engineering' is one in a series of Foresight Reports ${ }^{3}$ on themes where LRF see the need for further developments. The recommendations in the Foresight Reviews are followed up in practice through the funding of new programmes.

When catastrophic failures occur, lives can be lost, and damage may be unrecoverable. This is why the LRF has chosen resilience engineering as one of its research priorities. How can we build systems, infrastructures, networks, organizations and the associated human and social capacity to withstand stress and shock? How can we make catastrophic failures less likely when we do not always know what the risks are? The Resilience Shift project is all about answering these questions.

\subsection{Understanding the resilience landscape}

During 2017, the Resilience Shift funded a series of activities designed to inform direction setting and strategy, testing everything rigorously along the way. The programme has adopted a core principle to 'focus on the supply' side, meaning providing what is needed by the intended audience, as opposed to producing products where there may not be an established demand. Therefore, understanding the landscape was an essential activity.

There were 10 agenda setting publications produced by 10 grantees ${ }^{4}$. These covered a wide range of topics within infrastructure resilience, spanning from resilience return on investment, to water and flood resilience, to education for resilience and complex systems thinking.

\footnotetext{
2 https://www.arup.com/

${ }^{3}$ See LRF website for Foresight reports: https:/www.lrfoundation.org.uk/en/publications/

${ }^{4}$ To view the agenda setting research, please visit: https://www.resilienceshift.org/publication-category/agendasetting-research/
} 
The culmination of the 'agenda setting' work resulted in the publication of the 'Understanding the Landscape' report [4]. This report discusses the current state of practice regarding the resilience of infrastructure systems.

Some key findings, many of which are reflected in the wider literature, included:

- Resilience must move from theory to practice: despite a growing body of academic work, resilience must now move from theory to practice, building in learning loops to document, inform and improve education, practice and outcomes.

- There are numerous professional routes to resilience: we need to be mindful of building on best practice and specific sector expertise. However, the scope and parameters for infrastructure projects will need to broaden if we are to include resilience considerations from the outset.

- Good reasons exist for classic (reductionist) engineering approaches: that consider single assets, not systems, and require design to fixed thresholds. For many decades, these approaches were more than adequate, with the obvious evidence being that where best practices are followed, structures and infrastructure generally do not fail.

- Infrastructure design and management has traditionally been based on past events and performance: using sound statistical and probabilistic approaches based on historic observations, as well as modelling and expert opinion. The difference being that now we live in a much more complex, interdependent world and we must design for a diverse and uncertain future.

- An adaptive approach is needed in design and engineering practice: planning, design, construction and operation of critical infrastructure requires decision making in respect of multiple known and unknown hazards and highly uncertain costs.

- There are tensions challenging current practice: reflecting the debate around the differences between the definitions and evaluation of risk and resilience. Also, the challenge of managing risk to known hazards versus deep uncertainty and emerging risks.

\subsection{Vision}

The vision for the Resilience Shift is a safer and better world through resilient infrastructure. The programmes activities are planned and designed around six key 'impact statements' (see also Figure 1):

- A common understanding of sectors, including their supply chains and governance, as global systems and the effect of decisions within these systems on the resilience of society.

- Adoption of dynamic, performance-based (resilience based) design approaches in broad practice.

- Adoption or use of tools and processes to value resilience and make sure that value is realised throughout the project life cycle by project owners, developers, financiers and insurers.

- The use of integrated systems approaches as context for critical infrastructure systems.

- Integration of systems thinking and resilience concepts into the education and understanding of those responsible for planning, designing, delivering and operating critical infrastructure. 
- Adoption of transformative technologies that facilitate (rather than compromise) critical system functionality.

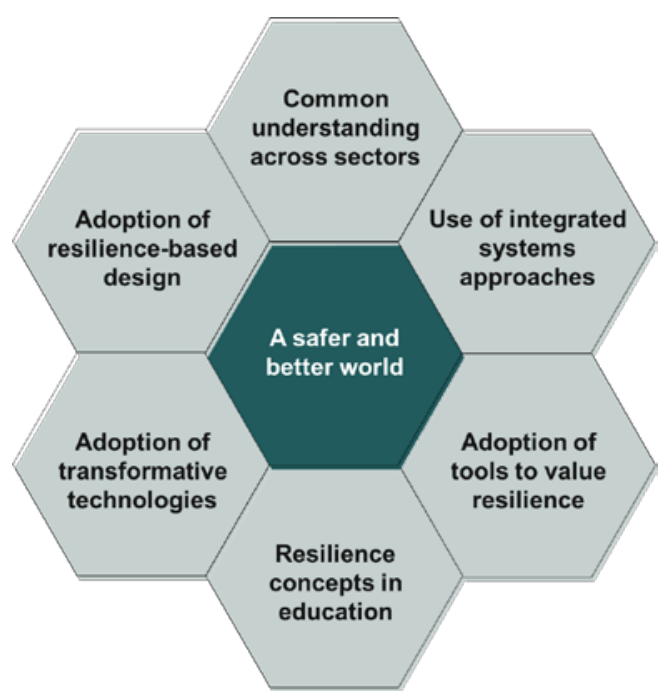

Figure 1: What success looks like for the Resilience Shift

The Resilience Shift wants to shift how critical infrastructure is financed, planned, designed, delivered and operated, to make it more resilient, to make sure the public gets the service it expects. The initiative wants to re-orient professional practice from a focus on infrastructure as an asset, to a focus on infrastructure as part of a system that provides services under both ordinary and extraordinary circumstances.

The focus of the Resilience Shift is to do work, and support others to do work, that will shift the worldwide approach to resilience in practice. The ultimate benefit of a 'resilience shift' will be greater security, and enhanced safety of life, property and the environment.

\subsection{Activities}

Our projects, investments and events are designed to contribute towards our impact and outcomes (Figure 1). Outputs ${ }^{5}$ to date have included knowledge resources, published materials, and the ongoing development of tools and primers.

We have focused our projects, following our agenda setting work in our first year, around three key areas that include:

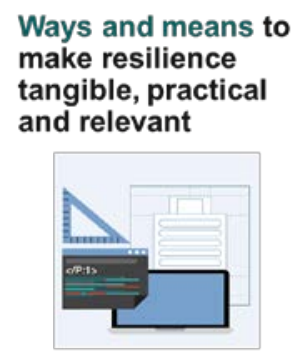

Focusing on ways to make resilience tangible, practical and relevant by equipping experts and decision makers with the tools, approaches, technology and educational practices needed to put resilience into practice.

This requires clarity on the key concepts that matter to professionals, be they designers, engineers, asset owners, investors or regulators.

\footnotetext{
${ }^{5}$ https://www.resilienceshift.org/publications/ 
Incentives driving resilience practice

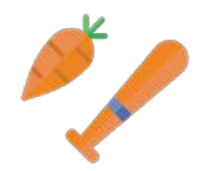

Transfering theory to practice within and between sectors

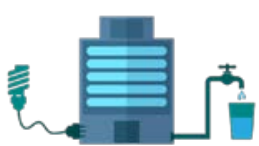

Focusing on incentivizing resilience. It acknowledges that the extent to which what infrastructure organisations ask for and what their supply chain can do depends strongly on incentives emerging from standards setting bodies, public policy including regulation, as well as from insurance and the finance industries, and the views of the public.

Focusing on applying resilience in practice within and between different sectors with sector leaders. This will allow us to test ideas about design and engineering to protect, provide and connect, rather than to build and operate a specific asset. We have selected the water sector for the first theory-to-practice partnership. 


\section{CURRENT PROGRESS}

Based on the agenda setting work, and the creation of the impact framework based on the expected outcomes defined in Figure 1, the Resilience Shift took the most promising ideas and turned them into a first round of projects. In year two (2018), the initiative has been further putting theory into practice. The following sections provide a summary of the work that the Resilience Shift and its grantees has been doing, covering the three key areas of the programme (as defined in Section 2.4).

\subsection{Collaboration}

The Resilience Shift believes that growing a community and sharing knowledge is critical to influencing others. To date the initiative has collaborated with over 30 grantees, from 12 countries $^{6}$. This has helped strengthen the programmes global network. External organisations can collaborate with the Resilience Shift through several means ${ }^{7}$.

Events play a key and exciting role in the Resilience Shift's collaborative approach. At the end of 2018, the initiative had hosted and attended 36 events $^{8}$, reaching out to over 1000 individuals (Figure 2). Recent events have included project-related workshops and three industry roundtables ${ }^{9}$ that have been instrumental in both building community, inspiring stakeholders about the need for a 'shift', validating our understanding of what is needed, and generating knowledge. Stakeholders have included representatives from industry bodies, government, global organisations, research institutes, non-profit organisations, specialist consultancies and infrastructure owners/operators.

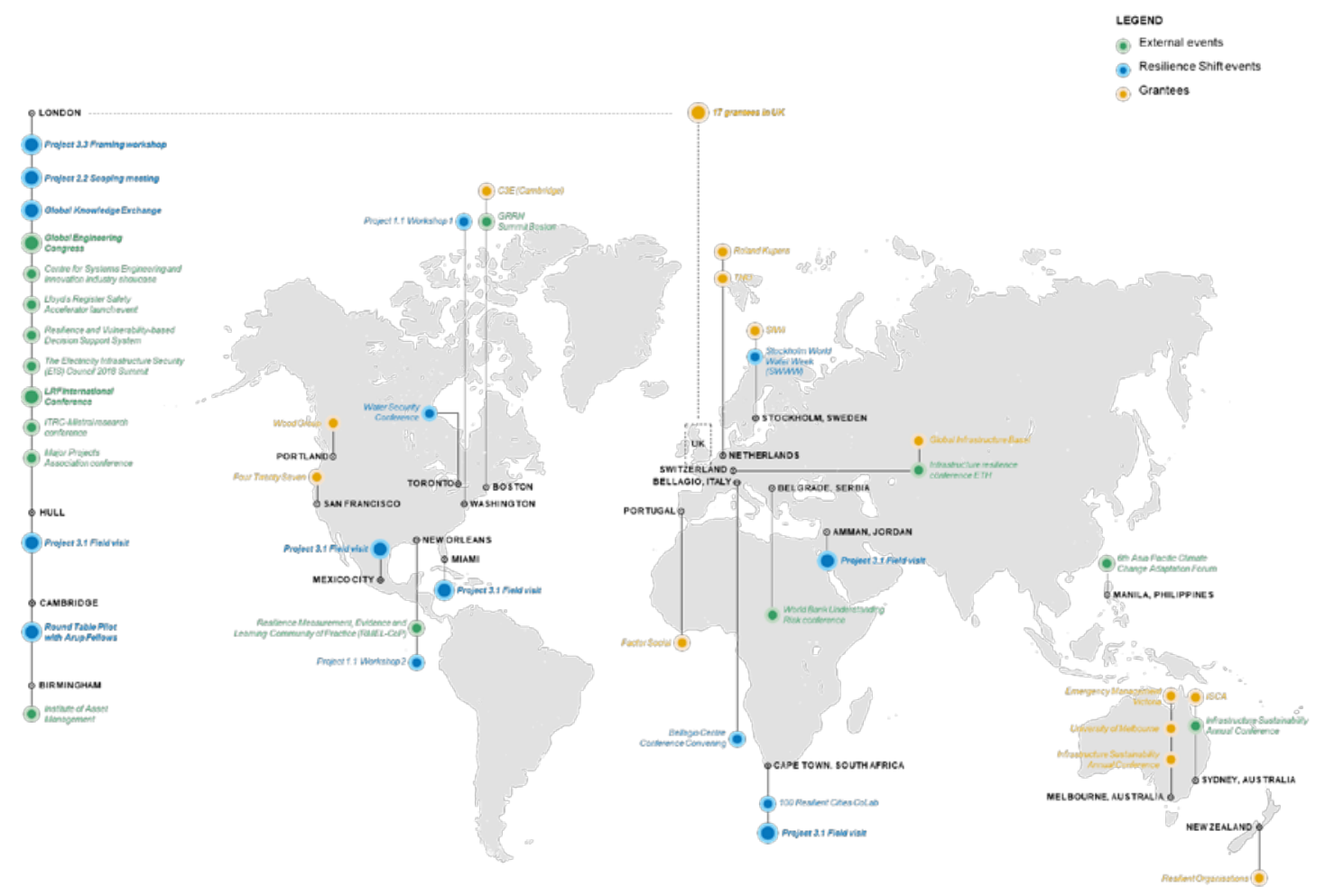

Figure 2: The Resilience Shift's external events attended, global events hosted and grantee locations (representative to end of 2018)

\footnotetext{
${ }^{6}$ https://www.resilienceshift.org/approach/collaborators/

${ }^{7}$ https://www.resilienceshift.org/work-with-us/

${ }^{8}$ https://www.resilienceshift.org/events/

${ }^{9}$ https://www.resilienceshift.org/activities/round-tables/
} 


\subsection{A value-based approach to critical infrastructure resilience}

One of the biggest challenges for critical infrastructure is breaking down the silos within and between infrastructure providers and customers, along the supply chain, so that everyone is focused on delivering resilience value where they can. The Resilience Shift has found that an infrastructure value chain (Figure $3^{10}$ ) is extremely useful for connecting the concepts of resilience and value in the context of the infrastructure lifecycle. It is also an approach that will be familiar to everyone working on the design, delivery and operation of infrastructure systems.

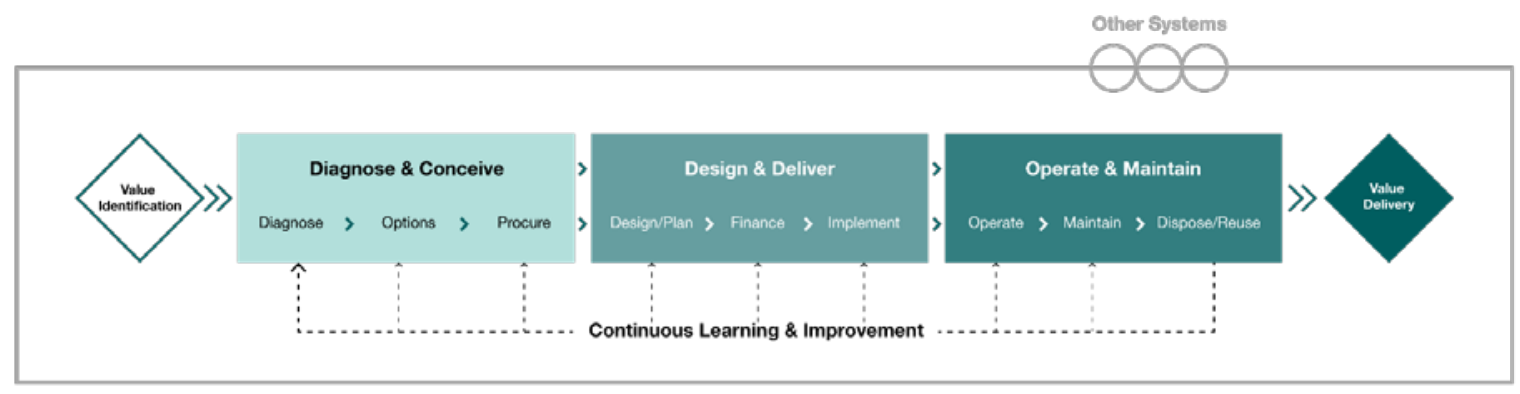

Figure 3: Infrastructure lifecycle value-chain

The concept of value chains is not new, and they are widely used across other industries [5]. This concept of joining the dots helps articulate the contribution of all parties in delivering the overall function and value of infrastructure systems and helps to align stakeholders behind a common outcome. We believe that a value chain approach to the resilience of critical infrastructure will help us to do this, through:

- Articulating why it is important that resilience value is created, enabled and protected at each stage and carried through the value chain because the resilience increases the value of the service provided, by reducing the impact of disruptions.

- To show how to 'do' resilience by mapping tools and approaches that can be used to enhance resilience at different parts of the value chain and at a level of sophistication appropriate for a stakeholders role.

- To indicate where the entry points and opportunities to create, enable and protect resilience value are for different stakeholders in the value chain and explain how they are connected.

\subsection{Ways and means to improve resilience in practice}

As part of the challenge to make resilience practical, tangible and relevant, work has been done to identify and assess the current range of tools and approaches that contribute to enhancing the resilience of critical infrastructure. This has already led to the collation and curation of more than 60 resilience tools and approaches. The Resilience Shift intends to share these tools and approaches through an accessible platform ${ }^{11}$, while also connecting relevant end-users to developers through a community of practice.

\footnotetext{
${ }^{10}$ See our website for more information and animation on the value chain concept: https://www.resilienceshift.org/approach/value-chain/

${ }^{11}$ Will be available on the Resilience Shift website in mid-2019.
} 
In order to begin developing a community of practice, workshops have been initially held in New Orleans [6], Washington DC [7] (Figure 4) and London [8]. These workshops were supported by the Resilience Shift's grantees, including: 100 Resilient Cities ${ }^{12}$, MMI Thornton Tomasetti ${ }^{13}$, The Schumacher Institute and Igor Linkov ${ }^{14}$. Bringing together tool users and developers, the workshops created an opportunity to explore the issues faced in the implementation of resilience tools

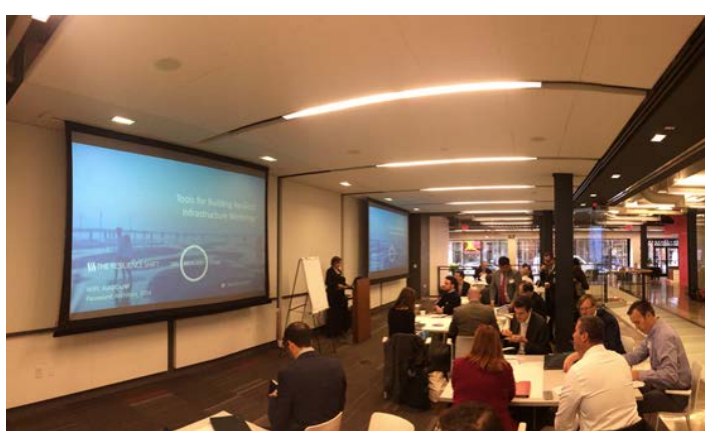

Figure 4: Workshop in Washington DC

(Figure 5). The workshops adopted the Action Learning methodology ${ }^{15}$ developed by the Schumacher Institute, to engage insightful thoughts from attendees [9].

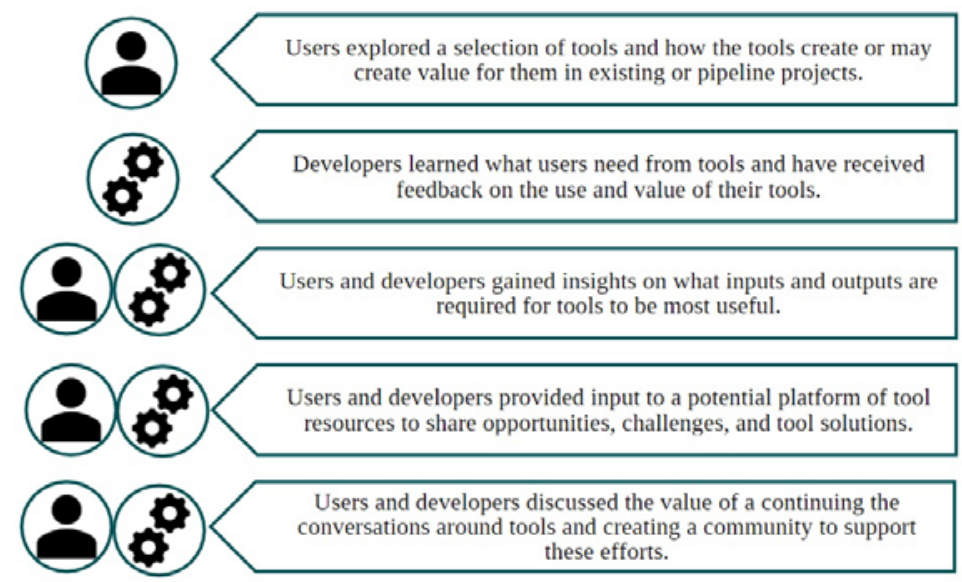

Figure 5: Approach taken in tools and approaches workshops

\subsection{Understanding the incentives}

Incentives that will drive changes in behavior towards more resilient critical infrastructure systems are emerging from standards setting bodies, public policy including regulation, as well as from insurance and the finance industries, and the views of customers. Organisations are often unaware of key incentives or other levers to embed and enhance resilience in the services that they provide to society. Moreover, there can be a lack of awareness of the value that resilience can bring, both to their own organisation and to others in the wider system-ofsystems.

The work of the Resilience Shift in this area has been focused on both understanding the policy and standards and their impact on delivering resilience, alongside understanding the incentives that drive resilience measures in different industries in practice. The role of public policy in critical infrastructure

\footnotetext{
${ }^{12}$ https://www.100resilientcities.org/

${ }^{13}$ https://www.mmiengineering.com/

${ }^{14}$ Affiliated with the US Army Corps of Engineers: https://www.erc Sheet-Article-View/Article/920870/risk-and-decision-science/

${ }^{15}$ https://www.resilienceshift.org/action-learning/
}

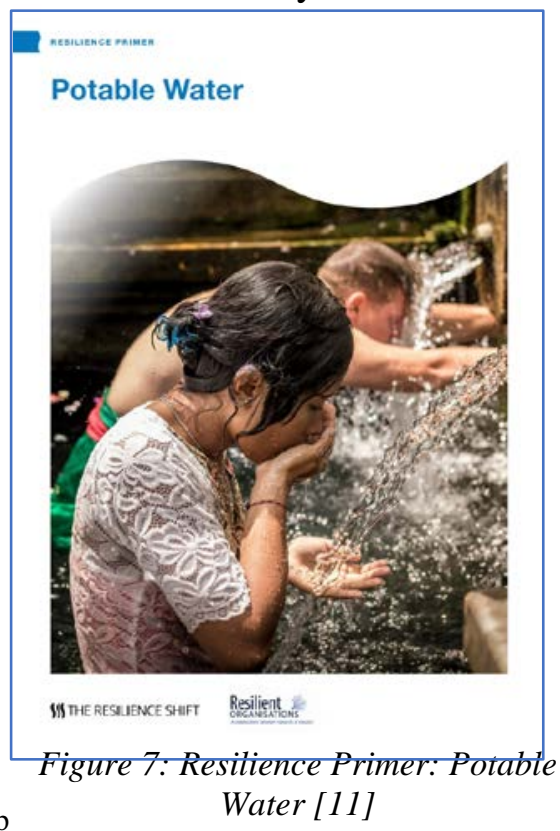


resilience is explored in further detail in a research report [10], available for download.

In 2018, four grantees ${ }^{16}$ were commissioned to develop industry-specific primers that will help key players understand what they can do differently to improve resilience in their industry. The primers will identify:

- Current best practices by leading organisations that embed resilience into their decisionmaking, and the incentives/levers that drive them.

- Incentives/levers that are currently available for driving resilience, but may not capitalized on due to lack of awareness of their existence.

- Knowledge of how to scale and augment the current menu of resilience incentives/levers.

Primers are being developed for the energy, ports, road, rail and water [11] sectors ${ }^{17}$. Development of these primers has been informed by an extensive review of relevant academic research, industry reports, policy papers, case studies and other resources as well as semistructured interviews with a select group of industry stakeholders and incentivisers.

\subsection{Putting theory into practice, initially in the water sector}

Deep dive activities funded by the Resilience Shift, that have focused on the water sector, have demonstrated the momentum that can be generated when multiple stakeholders are engaged in the 'co-creation' of a practical approach that can help to overcome barriers and start to put resilience into practice. During field visits undertaken in $2018^{18}$, more than $30 \%$ of survey respondents cited 'governance' as one of their biggest resilience challenges, and we believe this is true of other sectors.

To address this challenge, OurWater ${ }^{19}$ [12] has been developed to address the growing need for tools that can help cities share information between different stakeholders and visualize complex interactions. This goal of understanding the city's water landscape is one critical element of moving towards the goal of building cities' capacity to endure, adapt and transform in the face of water challenges. The report [12] and beta tool (April 2019) represents a collaborative effort between Arup $^{20}$ and the Stockholm International Water Institute ${ }^{21}$; with funding from the Resilience Shift.

\subsection{Round tables}

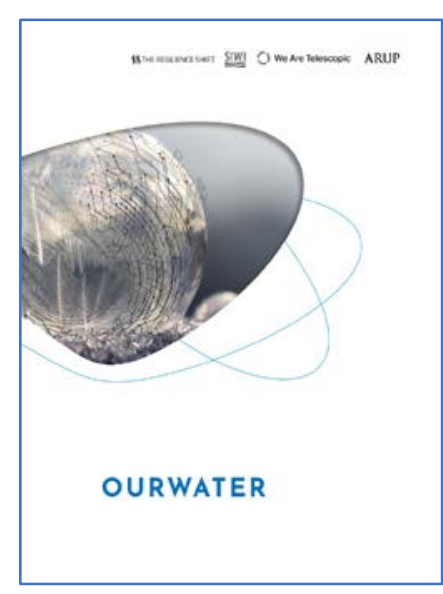

Figure 8: OurWater Report [12]

A series of round table workshops have been facilitated focusing on Ports (in London, UK), city-scale modelling (San Francisco, USA), and recovery from earthquake (Christchurch, New Zealand). Factual workshop reports are available for download on the Resilience Shift website. These events engaged with senior-level practitioners, with expertise associated with

\footnotetext{
${ }^{16}$ Including; Four Twenty Seven (http://427mt.com//); Resilient Organisations (https://www.resorgs.org.nz/),
TRL (https://trl.co.uk/), and; Wood (https://www.woodplc.com/).

${ }^{17}$ Due for publication by May 2019.

${ }^{18}$ For example, Cape Town: https://www.resilienceshift.org/publication/city-characterisation-report-cape-town/

${ }^{19}$ https://app.ourwater.city/

${ }^{20}$ https://www.arup.com/

${ }^{21}$ https://www.siwi.org/
} 
diverse aspects of critical infrastructure systems. The round tables provide the basis for crosssector learning and collaboration, creating a more informed view on interdependency, what is missing in governance frameworks and standards, and identifying measures to embed resilient design into modern infrastructure systems. They also provide participants with the opportunity to explore real challenges within their sector. The round table platform brings in other key stakeholders, encouraging inter-sector collaboration and communication. The round tables are designed to support the Resilience Shift activities described above across all workstreams.

\section{FUTURE PLANS}

Planned activities will continue, with the detailed planning of these activities currently being reviewed with the aim to incorporate the results achieved so far. New workshops and meetings are being planned and additional grants will be awarded in due course.

Some new themes have emerged during the first couple of years which call for closer attention. One example is the infrastructure vulnerability to shutdown of electric power supplies and the interdependencies of sectors. The Resilience Shift has partnered with the Electric Infrastructure Security Council ${ }^{22}$ to enhance the understanding of dependencies on electric power supply and cascading failures. We recently hosted together with the EIS Council, two 'black sky' EARTH EX resilience exercises, in London and Glasgow, to learn how to build better multi-sector resilience.

The Resilience Shift has commenced a project on how infrastructure in the global food supply chain is critical in ensuring that both food and materials/goods reach their markets. Failures at any point in a supply chain will have a cascading, potentially global, impact. A series of global workshops are being held on this subject in 2019. The project will also seek to work closer with private asset owners as it is evident that they are a key stakeholder in infrastructure management. Finance and insurance sectors are included in this list.

The Resilience Shift will continue to seek close engagement with public and private sector organisations engaged in financing, insuring, procuring, planning, designing, delivering, operating, maintaining critical infrastructure systems.

\section{CONCLUSIONS}

This report sets out the journey so far of the Resilience Shift in terms of its origins, objectives and findings and publications to date. The focus has been on:

- Enhancing the understanding of practitioners of what matters, and what drives best practice, in terms of the resilience of critical infrastructure systems, through research, guidance and convening events, and;

- On providing access to existing and emerging tools and approaches that will help put this into practice.

The emerging body of knowledge, tools and approaches that the Resilience Shift is producing, funding, and curating, will be available to download through www.resilienceshift.org. The sharing of results as they emerge is key principle of our way of working. This body of knowledge will also inform future programme-level decisions, understanding of the

\footnotetext{
${ }^{22}$ https://www.eiscouncil.org/
} 
incentives that exist and that are needed, and the opportunities for achieving impact and influence.

The project will continue with the same overall aims and objectives. The industry will be invited to compete for grants to be awarded as well as invited to workshops and presentations ${ }^{23}$.

\section{ACKNOWLEDGEMENTS}

The authors would like to recognise the valuable input from all of the Resilience Shift grantees and team members who have made this paper possible. We also thank Dr Nancy Kete, and Marcela Ruibal who introduced the 'value-based' approach, that continues to influence the initiative's work.

We also recognise the valuable input provided by Prof. Peter Guthrie and his team at Cambridge University. In particular, their role as a critical reviewer and discussion partner for the Programme Board and to the Resilience Shift team, including their organisation and chairing of the Round Table series.

\footnotetext{
${ }^{23}$ Please sign up to our blog to keep updated with programme news, including grant opportunities: https://www.resilienceshift.org/blog/ 


\section{REFERENCES}

[1] WEF (2019) The Global Risks Report 2019: $14^{\text {th }}$ Edition. Available at: http://www3.weforum.org/docs/WEF_Global_Risks_Report_2019.pdf

[2] Marchese D, Reynolds E, Bates ME, Morgan H, Clark SS, Linkov I (2018) Resilience and sustainability: Similarities and differences in environmental management applications. Science of the Total Environment. 613-614: 1275-1283.

[3] Lloyd's Register Foundation (2015) Foresight review of resilience engineering: Designing for the expected and unexpected. Available at: https://www.lrfoundation.org.uk/en/publications/resilience-engineering/

[4] Mian J, da Silva J, Kete N, Pritchard O, Borruel XA, Goode W (2017) Critical Infrastructure Resilience: Understanding the Landscape. Resilience Shift. Available at: https://www.resilienceshift.org/wp-content/uploads/2018/10/Critical-infrastructureresilience_RevA_Final_011018.pdf

[5] Kaplinsky R, Morris M (2001) A Handbook for Value Chain Research. Available at: https://www.researchgate.net/publication/42791981_A_Handbook_for_Value_Chain_Researc $\mathrm{h}$

[6] The Schumacher Institute/Global Infrastructure Basel (2019) Tools for resilient infrastructure: New Orleans Workshop. Available at:

https://www.resilienceshift.org/publication/tools-workshop-neworleans/

[7] 100 Resilient Cities (2019) Tools for Resilient Infrastructure: Washington DC Workshop. Available at: https://www.resilienceshift.org/publication/tools-workshop-washington/

[8] The Schumacher Institute (2019) Tools for Resilient Infrastructure: London Workshop. Available at: https://www.resilienceshift.org/publication/tools-workshop-london/

[9] MacLean M, Gill S (In Progress) Exploring challenges in the Implementation of Resilience. Submitted to REA Symposium.

[10] Keele S, Coenen L (2019) The role of Public Policy in Critical Infrastructure Resilience. Available at: https://www.resilienceshift.org/publication/role-of-public-policy/

[11] Hatton T, Kay E, Naderpajouh N, Aldrich D (2019) Resilience Primer: Potable Water. March 2019. Available at: https://www.resilienceshift.org/wpcontent/uploads/2019/04/RP_Potable-Water_FINAL-Pages.pdf

[12] Arup \& SIWI (2019) OurWater. Available at: https://www.resilienceshift.org/publication/watershare/ 